\title{
Prophylaxis of Postoperative Acute Endopthalmitis Following Cataract Surgery
}

\author{
Khalifa Alsawidi1 ${ }^{*}$, Hamdy Abdulhady2 ${ }^{2}$, Miriam Benlashhar ${ }^{1}$, Amal Ahmed ${ }^{1}$ \\ ${ }^{1}$ Tripoli Eye Hospital, Tripoli, Libya \\ ${ }^{2}$ Abdulhady Eye Clinic, Tripoli, Libya \\ Email: ${ }^{\star}$ KhalifaAlsawidi@gmail.com
}

How to cite this paper: Alsawidi, K., Abdulhady, H., Benlashhar, M. and Ahmed, A. (2020) Prophylaxis of Postoperative Acute Endopthalmitis Following Cataract Surgery. Open Journal of Ophthalmology, 10, 28-32. https://doi.org/10.4236/ojoph.2020.101004

Received: November 7, 2019

Accepted: December 27, 2019

Published: December 30, 2019

Copyright $\odot 2020$ by author(s) and Scientific Research Publishing Inc. This work is licensed under the Creative Commons Attribution International License (CC BY 4.0).

http://creativecommons.org/licenses/by/4.0/

\begin{abstract}
Background: Postoperative Endophthalmitis is a rare complication after intraocular surgeries. However, it remains one of the most devastating complications following cataract surgery because of its poor prognosis. We describe the effects of antibiotic prophylaxis on the incidence of postoperative endophthalmitis after cataract surgery, particularly phacoemulsification. Aim of the work: To identify risk factors of acute endophthalmitis and describe the effects of antibiotic prophylaxis on the incidence of postoperative endophthalmitis after phacoemulsification surgery. Methods: A prospective randomized cataract surgery study recruited 300 patients; 150 patients who received just intracameral vigamox at the end of surgery without topical antibiotics before surgery and 150 patients who received both intracameral vigamoxas well as topical perioperative vigamox which either just half an hour before surgery or three days preceding surgery. We took a conjunctival swab before and after vigamox administration in order to detect the type of conjunctival flora in the Libyan population as well as the effect of the vigamox on the conjunctival flora by using Phoenix-BD machine. In total we collected 166 specimens (83 patients). Results: In all groups no acute post-operative endophthalmitis was reported. Conclusions: The study was based on intracameral Moxifloxacin at the end of surgery with and without topical antibiotics drops administration. The incidence of postcataract endophthalmitis was not reported in our study after using intracameral antibiotics, as it seems to be effective in preventing endophthalmitis after cataract surgery.
\end{abstract}

\section{Keywords}

Prophylaxis, Postoperative Acute Endopthalmitis, Cataract Surgery

\section{Introduction}

Endophthalmitis following cataract surgery is rare but continues to be dreaded 
complication following cataract surgery. Over the years, the incidence of endophthalmitis has declined because of various preoperative and perioperative measures. They include use of antiseptic and appropriate surgical draping techniques. Acute postoperative endophthalmitis is generally defined as an inflammation involving both the anterior and posterior segments of the eye after intraocular surgery occurring within six weeks of the surgery. It is most often caused by bacteria, fungi or rarely parasites. It might be due to microbial contamination spreading from the ocular surface or open incision (wound) or contaminated instruments or intraocular lenses (IOLs).

The Endophthalmitis Vitrectomy Study (EVS) demonstrated that most isolates causing clinical endophthalmitis are introduced into the eye from the patient's conjunctival flora [1].

However, contamination of sterilized instruments, disposable supplies, prepared solutions, surgical field, or the intraocular lens all has been reported. Epidemic clusters of endophthalmitis have resulted from these types of external contaminations [2] [3].

The current treatment approach in general follows the findings of the EVS study, with initial vitreous tap and antibiotic injection for most cases, and early vitrectomy for cases in which the vision is LP.

The occurrence, severity and clinical course of endophthalmitis depends on the route of infection, the virulence and number of inoculated pathogens as well as the patient's immune state and the time of examination [4]. Although in 29 to 43 per cent of cataract operations, intraocular contamination occurs with facultative pathogenic bacteria from the ocular surface, the development of endophthalmitis does not occur [5].

The incidence of postcataractsurgery endophthalmitis varies, ranging from $<0.05 \%$ to $>0.3 \%$ [6]. This range in the incidence of infection appears to be consistent across numerous patient populations from all over the world [7].

The incidence of acute postoperative endophthalmitis after cataract surgery in the Tripoli Eye Hospitals was in $2008(0.35 \%)$ which increased markedly in comparison to the year $2007(0.16 \%)$.

There is a debate over the most appropriate strategy for preventing infection around the time of intraocular surgery. Most ophthalmologists agree that several steps are important in reducing the rate of infection, including minimizing the ocular surface flora with $5 \%$ povidone-iodine, keeping the eyelashes covered, and a careful and aseptic surgical technique with appropriate wound closure. Some surgeons advocate the addition of broad-spectrum topical antibiotics for either three days or just half hour prior to the surgery to reduce surface pathogens, although this recommendation remains controversial.

A large European study (ESCRS) found that intracameral cefuroxime given at the time of cataract surgery reduced the rate of postoperative endophthalmitis [6].

Results of the ESCRS postoperative endophthalmitis study, the Endophthalmitis Vitrectomy Study (EVS) and other studies assessing the causative organism 
demonstrate that Gram-positive organisms account for $90 \%$ or more of pathogens isolated in culture-positive cases of postoperative endophthalmitis following cataract surgery, with coagulase-negative Staphylococci (i.e., Staphylococcus epidermidis) and Staphylococcus aureus representing the leading causes [6].

\section{Aim of the Study}

To identify risk factors of acute endophthalmitis and describe the effects of antibiotic prophylaxis on the incidence of postoperative endophthalmitis after phacoemulsification surgery.

\section{Methods}

A prospective randomized cataract surgery study recruited 300 patients. All surgeries were performed at Hamdy Eye Clinic by the same surgeon. The study was based on intracameral vigamox (Moxifloxacin, $4^{\text {th }}$-generation fluoroquinolones) 50 microgram in $0.1 \mathrm{ml}$ at the end of surgery with and without topical vigamox drops administration.

The study included 150 patients who received just intracameral vigamox at the end of surgery without topical antibiotics before surgery. The second group another 150 patients who received both intracameral vigamox as well as topical perioperative vigamox which either just half an hour before surgery or three days preceding surgery. The first group included 75 patients where vigamox drops were administered as at 5-minute intervals commencing immediately 30 minutes before surgery. The second group 75 patients were received vigamox prescribed 4 times daily for three days preceding the surgery. In this group we took a conjunctival swab before and after vigamox administration in order to detect the type of conjunctival flora in the Libyan population as well as the effect of the vigamox on the conjunctival flora by using Phoenix-BD machine. In total we collected 166 specimen ( 83 patients).

All patients were prepared with povidone-iodine 5\% drops 3 minutes before surgery The study evaluated the role of pre-operative antibiotics, which are normally administered anywhere between 30 minutes to three days before surgery. All instruments for surgery were sterile and care was carried out with both washing the instruments and autoclaving them. Bottles of solution containing BSS (balanced salt solution) were never be kept or used for more than one operating session. The comparison of treatment effects and other risk factors were estimated.

Inclusion criteria were cataract surgery which was done by phacoemulsification only.

We exclude traumatic cataract, children as well as other combined surgical procedure which might be done with phacoemulsification such as trabeculectomy, vitrectomy or avast in injection.

\section{Results}

Followed them up for 9 months to exclude acute as well as chronic endophthal- 
mitis. Patients were 80 females and 70 males. In all groups no acute post operative endophthalmitis was reported.

\section{Discussion}

Intracameralvigamox alone is very effective method in prevention of acute post operative endophthalmitis. Although administration of topical antibiotics showed marked reduction of the patients conjunctival flora, it did affect the incidence of occurrence of acute postoperative endophthalmitis in the patients who just received intracameral vigamox only.

Intracameralvigamox is superior to intracameral cefuroxime injection because intracameral antibiotic mixtures were a significant concern to surgeons. These risks might include dilution errors, bacterial contamination. Indeed those concerns are not applicable to vigamox because you don't have to dilute it and just take $0-1 \mathrm{ml}$ from the bottle and immediately injected into the anterior chamber.

\section{Conclusion}

There is a strong effect intracameral vigamox on the incidence of acute postoperative endophthalmitis following phacoemulsification. Vigamox administration didn't cause any serious complications such as corneal endothelium cell loss or toxic anterior segment syndrome.

\section{Acknowledgements}

Authors would like to acknowledge the Libyan National Agency for scientific research for their support and help to carry out the research.

\section{Conflicts of Interest}

The authors declare no conflicts of interest regarding the publication of this paper.

\section{References}

[1] Endophthalmitis Vitrectomy Study Group (1996) Microbiologic Factors and Visual Outcome in the Endophthalmitis Vitrectomy Study. American Journal of Ophthalmology, 122, 830-846. https://doi.org/10.1016/S0002-9394(14)70380-0

[2] Gibb, A.P., Fleck, B.W. and Kempton-Smith, L. (2006) A Cluster of Deep Bacterial Infections Following Eye Surgery Associated with Construction Dust. Journal of Hospital Infection, 63, 197-200. https://doi.org/10.1016/j.jhin.2006.01.010

[3] Cruciani, M., Malena, M., Amalfitano, G., et al. (1998) Molecular Epidemiology in a Cluster of Cases of Postoperative Pseudomonas aeruginosa Endophthalmitis. Clinical Infectious Diseases, 26, 330-333. https://doi.org/10.1086/516292

[4] Peyman, G., Lee, P. and Seal, D.V. (2004) Endophthalmitis: Diagnosis and Management. Taylor \& Francis, London, 1-270. https://doi.org/10.3109/9780203017630

[5] Sherwood, D.R., Rich, W.J., Jacobs, J.S., Hart, R.J. and Fairchild, Y.L. (1989) Bacterial Contamination of Intraocular and Extraocularfluids during Extracapsular Cataract Extraction. Eye, 3, 308-312. https://doi.org/10.1038/eye.1989.44 
[6] Endophthalmitis Study Group (2007) Prophylaxis of Postoperative Endophthalmitis Following Cataract Surgery: Results of the ESCRS Multicenter Study and Identification of Risk Factors. Journal of Cataract \& Refractive Surgery, 33, 978-988.

https://doi.org/10.1016/j.jcrs.2007.02.032

[7] Fintelmann, R.E. and Naseri, A. (2010) Prophylaxis of Postoperative Endophthalmitis Following Cataract Surgery: Current Status and Future Directions. Drugs, 70, 1395-1409. https://doi.org/10.2165/11537950-000000000-00000 